\title{
Equipamento e fármacos de emergência nos Cuidados de Saúde Primários
}

Ângela Cerqueira, ${ }^{*}$ Rui Gomes, ${ }^{* *}$ Marta Marquês, ${ }^{* * *}$ Marta Fragoeiro***

\section{RESUMO}

O Médico de Família necessita de ter a formação e o material adequados para poder actuar em situações de emergência na sua Unidade de Saúde e na prestação de cuidados na comunidade. Todas as unidades de saúde devem dispor de uma mala ou carro de emergência, com material e utilização reservados a estas situações. É da responsabilidade dos profissionais de cada unidade de saúde a elaboração dessa mala.

Este artigo pretende apoiar os profissionais na sua elaboração, revendo o equipamento e fármacos que devem ser incluídos na mala e respectiva operacionalização.

Palavras-Chave: Emergências; Cuidados de Saúde Primários; Equipamento; Fármacos.

\section{INTRODUÇÃO}

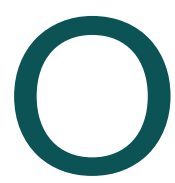

Médico de Família necessita dos conhecimentos, aptidões, equipamentos e fármacos adequados para a abordagem de situações de emergência na sua Unidade de Saúde ou quando presta cuidados na comunidade. Idealmente, todas as Unidades de Saúde deveriam ter equipamento e fármacos adequados para uma actuação rápida e eficaz nestas situações e a equipa de saúde devia estar familiarizada com o respectivo protocolo de utilização.

O equipamento de reanimação pode ser guardado numa sala de reanimação ou, mais frequentemente, numa mala que se transporta para o local da reanimação. É da responsabilidade dos profissionais da Unidade de Saúde organizar essa mala de emergência e respectiva manutenção. Esta mala deve estar selada e ser aberta apenas em situações de emergência ou para verificação e manutenção do material e terapêutica. A mala deve ser de transporte fácil e com vários compar-

\footnotetext{
*Médica de Família na Unidade Saúde Familiar de Tornada; Tripulante da Viatura Médica de Emergência e Reanimação do Centro Hospitalar Oeste Norte

**Médico de Medicina Interna na Unidade de Cuidados Intensivos do Hospital Garcia de Orta; Tripulante da Viatura Médica de Emergência e Reanimação do Hospital Garcia de Orta

***Médica de Família na Unidade Saúde Familiar da Cova da Piedade, ACES Almada
}

timentos permitindo o acesso rápido ao seu conteúdo. Os dispositivos e fármacos escolhidos devem ser adequados às situações emergentes mais frequentes, ao grau de treino dos profissionais de saúde e ao nível de intervenção desejado. Devem ser escolhidos itens que sejam ao mesmo tempo de fácil utilização e de fácil manutenção.

Todos os profissionais da Unidade de Saúde devem conhecer a localização do equipamento, o seu conteúdo e ter treino na sua utilização. Deve ainda existir um profissional nomeado com a responsabilidade de verificar a disponibilidade, adequabilidade e estado de conservação de todo o equipamento, idealmente uma vez por semana. $^{1}$

Este profissional deve ser ainda responsável, após cada utilização, pela verificação e reposição do material, confirmação da elaboração da folha de registo de selagem do carro, por selar novamente o carro e por verificar a elaboração do relatório escrito do evento de emergência.

Infelizmente, são ainda muitas as Unidades de Saúde que não dispõem do material necessário para situações de urgência/emergência, porém compete aos profissionais solicitar junto das entidades responsáveis o seu fornecimento de forma a prestar melhores cuidados de saúde à sua população numa situação emergente. 


\section{EQUIPAMENTO PARA A ABORDAGEM DE EMERGÊNCIAS}

Para a abordagem diagnóstica e monitorização terapêutica de situações emergentes, é fulcral a existência na Unidade de Saúde, de um monitor que permita a monitorização contínua de ritmo cardíaco, frequência cardíaca, tensão arterial, frequência respiratória e oximetria de pulso.

A desfibrilhação precoce é um dos elos da cadeia de sobrevivência, pelo que seria desejável a existência de um desfibrilhador.

Os desfibrilhadores automáticos externos são equipamentos desenvolvidos para a utilização por leigos com formação em suporte básico de vida. São mais baratos, fáceis de utilizar e mais seguros que os desfibrilhadores convencionais. Estas características podem facilitar a instituição generalizada de desfibrilhadores nas Unidades de Saúde, bem como a sua utilização por profissionais de saúde que por norma têm pouca experiência nestas situações clínicas. Existem modelos com monitor

\section{QUADRO I. Mala de Emergência - um exemplo do que deve conter $1,2,3,4,5,6$}

\begin{tabular}{|c|c|c|c|c|}
\hline Fármacos ev & Fármacos po & Outros & Material & $\begin{array}{l}\text { Equipamento de apoio à } \\
\text { Mala de Emergência }\end{array}$ \\
\hline $\begin{array}{l}\text { Adrenalina } \\
\text { Amiodarona } \\
\text { Aminofilina } \\
\text { Atropina } \\
\text { Clemastina } \\
\text { Diazepam } \\
\text { Dinitrato de isosorbido } \\
\text { Furosemida } \\
\text { Glicose } 30 \% \\
\text { Hidrocortisona } \\
\text { Hidroxizina } \\
\text { Prednisolona } \\
\text { Metilprednisolona } \\
\text { Metoclopramida } \\
\text { Morfina }\end{array}$ & $\begin{array}{l}\text { Aspirina } \\
\text { Captopril } \\
\text { Diazepam } \\
\text { Hidroxizina } \\
\text { Nitroglicerina }\end{array}$ & $\begin{array}{l}\text { Água destilada } \\
\text { Brometo ipratrópio } \\
\text { Budesonido inalado } \\
\text { Carvão activado } \\
\text { Dextrose } 5 \% \text { em água } \\
\text { Diazepam rectal } \\
\text { Oxigénio em bala } \\
\text { portátil } \\
\text { Salbutamol (inalador/ } \\
\text { /solução nebulização) } \\
\text { Soro fisiológico }\end{array}$ & $\begin{array}{l}\text { Adesivos } \\
\text { Agulhas ev, sc, im, } \\
\text { Álcool } \\
\text { Betadine dérmico, } \\
\text { Câmara expansora } \\
\text { Catéteres } 16 \text { a } 24 \\
\text { Compressas } \\
\text { Contentor cortantes } \\
\text { Esfingomanómetro e } \\
\text { braçadeiras adulto } \\
\text { e pediátrica } \\
\text { Estetoscópio } \\
\text { Garrote } \\
\text { Lâminas } \\
\text { Luvas } \\
\text { Máscara facial (O }) \\
\text { Medidor de glicemia } \\
\text { com tiras e lancetas } \\
\text { Óculos nasais } \\
\text { Pinça Magill } \\
\text { Seringas } \\
\text { Seringa insulina } \\
\text { Sistema soro } \\
\text { Sondas ENG } 16 \text { e } 18 \\
\text { e sacos colectores } \\
\text { Tesoura e Gillete } \\
\text { Tubos Guedel } \\
\text { Selos de substituição } \\
\text { (Laringoscópio, } \\
\text { Tubos EOT) }\end{array}$ & $\begin{array}{l}\text { Aspirador de secreções e sondas de } \\
\text { aspiração } \\
\text { DAE (e eléctrodos) } \\
\text { Electrocardiografo (e eléctrodos) } \\
\text { Insuflador manual e filtros/ } \\
\text { /máscara facial adulto e pediátrico } \\
\text { Monitor de PA, FC, FR e oximetria } \\
\text { de pulso }\end{array}$ \\
\hline
\end{tabular}

Legenda: ev: endovenosa; im: intra-muscular; sc: subcutânea; ENG: entubação naso-gástrica; EOT: entubação oro-traqueal; DAE: desfibrilhador automático externo; PA: pressão arterial; FC: frequência cardíaca; FR: frequência respiratória. 
analógico que permitem o reconhecimento do ritmo cardíaco, no entanto, este facto não dispensa a existência de um monitor que permita a monitorização contínua de parâmetros vitais e ritmo cardíaco.

Em situações de paragem cardio-respiratória, o insuflador manual, uma máscara facial adaptável e o tubo de Guedel são os requisitos mínimos para a abordagem da via aérea. A entubação oro-traqueal é a técnica de referência, mas tem uma curva de aprendizagem longa e requer experiência por parte de quem a realiza, sendo portanto, raramente exequível nos cuidados de saúde primários. Existem outras alternativas, como o Combitubo, o tubo laríngeo ou a máscara laríngea, que podem permitir uma melhor abordagem da via aérea e cujo manuseio é mais simples.

É igualmente importante existir uma marquesa ou tábua dura que permita um suporte básico de vida adequado na paragem cardio-respiratória. Em último caso, o chão pode servir como alternativa aceitável para esse fim, embora não constitua a solução ideal. ${ }^{2}$

No Quadro 1 consta um exemplo do que deve conter uma mala de emergência.

Uma Unidade de Saúde rural, longe de um hospital e sem acesso a serviços de emergência (VMER) pode ter de dispor de mais equipamento e treino do que as unidades urbanas. Além disto, deve sempre ser pesado o valor da estabilização da vítima versus o risco do atraso na chegada ao hospital.

\section{CONCLUSÃO}

Este artigo procura ilustrar o que deve ser o conceito de mala de emergência no âmbito da realidade clínica dos Cuidados de Saúde Primários. É responsabilidade dos profissionais o assegurar da sua existência e manutenção, as- sim como da formação contínua na área da emergência, adequada ao nível de cuidados que prestam.

Não é demais salientar a importância do médico de família, e de todos os profissionais numa Unidade de Saúde dos Cuidados de Saúde Primários, no assegurar dos elos da cadeia de sobrevivência, com todo o benefício conhecido para a sobrevida e qualidade de vida posterior das vítimas.

\section{REFERÊNCIAS BIBLIOGRÁFICAS}

1. Colquhoun M, Gabbott D, Mitchell S. Cardiopulmonary Resuscitation Guidance for clinical practice and training in Primary Care [Internet]. London: Resuscitation Council; 2001 Jul [acedido a 10/03/2010]. 13 p. Disponível em: http://www.resus.org.uk/pages/cpatpc.htm\#app2.

2. Committee on Pediatric Emergency Medicine. Preparation for emergencies in the offices of pediatricians and pediatric primary care providers. Pediatrics 2007 Jul; 120 (1): 200-12.

3. Handley AJ, Koster R, Monsieurs K, Perkins GD, Davies S, Bossaert L; European Resuscitation Council. European Resuscitation Council Guidelines for Resuscitation 2005: Section 2: Adult basic life support and use of automated external defibrillators. Resuscitation 2005 Dec; 67 Suppl 1: S7-23.

4. Nolan JP, Deakin CD, Soar J, Böttiger BW, Smith G, European Resuscitation Council. European Resuscitation Council Guidelines for Resuscitation 2005: Section 4: Adult advanced life support. Resuscitation 2005 Dec; 67 Suppl 1: S39-86.

5. Baird A. Emergency drugs in general practice. Aust Fam Physician 2008 Jul; 37 (7): 541-7.

6. INEM - Departamento de Formação em Emergência Médica. Manual de Suporte Avançado de Vida. Lisboa. INEM; 2007.

7. Endom EE. Preparing an office practice for pediatric emergencies. In: Basow DS, editor. Waltham, MA: UpToDate; 2010.

Os autores declaram não possuir conflitos de interesses.

ENDEREÇO PARA CORRESPONDÊNCIA
Ângela Cerqueira
Rua Poços das Caldas, N. 1 - $3 .{ }^{\circ}$ Andar
2500-290 Caldas da Rainha
E-mail: angelacerqueira@gmail.com

Ângela Cerqueira

2500-290 Caldas da Rainha

E-mail: angelacerqueira@gmail.com

\section{ABSTRACT}

\section{RESUSCITATION EQUIPMENT IN PRIMARY CARE}

The family doctor needs proper training and access to adequate material to be able to act in emergency situations in his healthcare practice or while delivering care in the community.

All healthcare practices should have an emergency box, or car, with use reserved to this kind of situations. It is the health professional's responsibility the putting together of this box. This article intends to support health professionals in the making of this emergency box, reviewing the equipment and drugs which should be included and the operationalization of the box.

Keywords: Emergencies; Primary Health Care; Equipment; Drugs. 